\title{
Using Laboratory Data to Aid Early Warning in Prospective Influenza Mortality Surveillance
}

\author{
Aye M. Moa*, David J. Muscatello, Robin Turner and C Raina Maclntyre
}

The University of New South Wales, Sydney, NSW, Australia

\section{Objective}

To demonstrate use of routine laboratory-confirmed influenza surveillance data to forecast predicted influenza-attributable deaths during the current influenza season. We also assessed whether including information on influenza type produced better surveillance forecasts.

\section{Introduction}

Several countries prospectively monitor influenza-attributable mortality using a variation of the Serfling seasonal time series model that uses sinusoidal terms for seasonality. ${ }^{1-4}$ Typically, a seasonal model from previous years is used to forecast current expected mortality. Using laboratory surveillance time series data in the model may enhance interpretation of the surveillance information.

\section{Methods}

We fit a Serfling-type, robust linear regression, time series model ${ }^{5}$ to weekly, all-age counts of influenza and pneumonia deaths for Australia, 2007-2011. Weekly laboratory-confirmed influenza counts were included as covariates; one model using total influenza was compared with a model including influenza type A and B. The two model forecasts of weekly deaths during 2012 were compared against observed deaths using root mean squared error (RMSE). An indicator variable was used to adjust for inflated testing during the 2009 pandemic year and laboratory data was lagged by two weeks.

\section{Results}

Both models provided a reasonable forecast for 2012 (Figures 1 and 2). RMSE for the 2012 forecasts were 12.08 and 9.37, for the total influenza and type A and B models, respectively; the influenza type $\mathrm{A}$ and $\mathrm{B}$ model had a better fit. The total influenza model predicted that an increase of 100 total influenza notifications in a week was associated with an increase of 0.4 (95\% CI: 0.02-0.9) deaths two weeks later. The influenza type A and B model predicted that an increase of 100 type A notifications in a week was associated with an increase of 1.2 (95\% CI: 0.7-1.8) deaths two weeks later. However, parameter estimate for influenza type $\mathrm{B}$ was negative.

\section{Conclusions}

We demonstrated a laboratory data-based time series model that may improve prospective mortality surveillance to assess the current season's influenza impact. The model would allow week to week forecast of expected deaths as new laboratory data is received. Also, the observed deaths could be compared with the forecast influenzaattributable deaths and if the observed deaths were higher than the forecast by a threshold amount, then we could signal a more virulent influenza strain or a more susceptible population than expected. Some statistical challenges remain.
Fig 1. Predicted, forecast and observed influenza and pneumonia deaths using the total influenza model, Australia, 2007- 2012

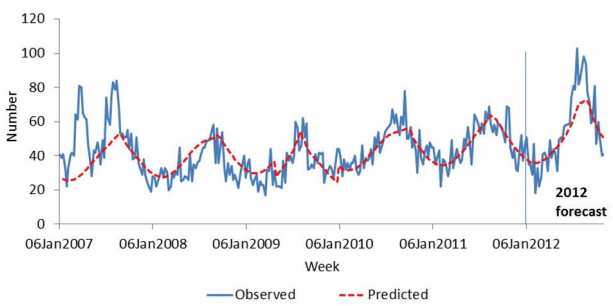

Fig 2. Predicted, forecast and observed influenza and pneumonia deaths using the influenza type A and B model, Australia, 2007- 2012

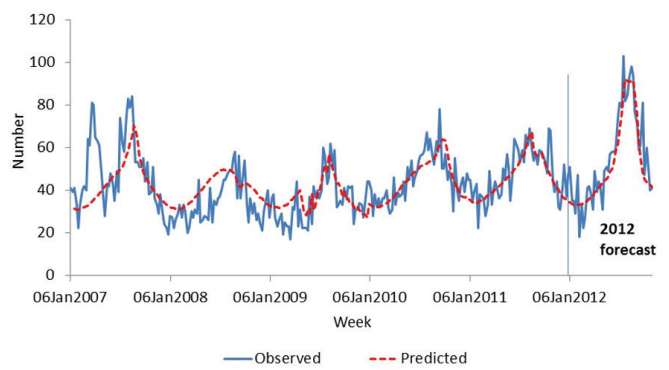

Keywords

influenza; surveillance; statistical models; mortality

\section{Acknowledgments}

Data were provided by the Australian Institute of Health and Welfare and the Australian Department of Health.

\section{References}

1. The Australian Department of Health. Australian influenza surveillance report and activity updates, 2015. Available from http://www.health. gov.au/flureport\#current. Accessed 26 August 2015.

2. Centers for Disease Control and Prevention. Weekly U.S. influenza surveillance report, 2015. Available from http://www.cdc.gov/flu/ weekly/. Accessed 26 August 2015.

3. Mortality monitoring in Europe. European mortality bulletin week 33, 2015. Available from http://www.euromomo.eu/. Accessed 26 August 2015.

4. Serfling R. Methods for Current Statistical Analysis of Excess Pneumonia-influenza Deaths. Public Health Reports 1963; 78(6): 494-506.

5. Muscatello DJ, Morton PM, Evans I, Gilmour R. Prospective surveillance of excess mortality due to influenza in New South Wales: feasibility and statistical approach. Communicable Diseases Intelligence Quarterly Report 2008; 32(4): 435-42. 